\title{
Thematic Issue: Driving Automation and Autonomy
}

\author{
Neville A Stanton
}

Human Factors Engineering, Transportation Research Group, Boldrewood Innovation Campus, Civil, Maritime and Environmental Engineering, Faculty of Engineering and Physical Sciences, University of Southampton, Burgess Road, Southampton, S016 7QF, UK.

\begin{abstract}
Automated driving has the potential to support drivers, freeing them up to do other things, such as work, rest or play. The problem is that in the present instantiation, automated driving requires the driver to perform a monitoring function and be ready to intervene if required. This is the worst of all worlds of automation. The monitoring task can be (if performed properly) more demanding than manual driving and the driver is not freed up to do other things. Worse still, is that the monitoring task cannot be sustained for long and, on occasions, led to a vehicle collisions because the driver cannot intervene in a timely manner. One of the first studies conducted over twenty years shows this to be the case and there really have not been any improvements since. This special issue reports on the latest development in vehicle automation and points to future directions that research should be directed.
\end{abstract}

\section{Relevance to human factors/Relevance to ergonomics theory}

Driving Automation and Autonomy is already upon us and the problems that were predicted twenty years ago are beginning to appear. These problems include shortfalls in expected benefits, equipment unreliability, driver skill fade, and error-inducing equipment designs. In addition, the driver becomes both physically and mentally detached from the task of driving and may engage with other non-driving tasks. Ironically, if the driver does not engage with other tasks then can suffer from reduced attentional resources (making them less able to regain control from the vehicle in an emergency). If the driver does engage with other non-driving tasks then their attentional resource pool do not deplete to the same extent (offering a protective effect) but the distraction of the other task(s) can slow down the reclaim of vehicle control from automation. This is one of the main dilemmas with automated driving, which the papers in this special issue address to a greater or lesser extent.

\section{Introduction to Driving Automation and Autonomy}

One of the main drivers for automated and autonomous driving is the potential for betterment of the human condition. Potentially at least, autonomous driving systems could support elderly, impaired, young, busy and bored drivers. Further, these autonomous driving systems might also be able to reduce societal problems, such as congestion, collisions and emissions. At present, however, we are some distance away from driving systems that can completely remove the need for human oversight, monitoring and intervention. In fact, Bainbridge's (1983) ironies of automation are as problematic today for vehicle automation as they were for industrial and flight-deck automation 35 years ago. Bainbridge's 
ironies included: tasks left over, de-skilling, out-of-the-loop, and monitoring. Traditionally, automation has been designed in a techno-centric manner, without proper consideration to the role of humans (drivers in the case of the special issue), which in turn has given rise to the problems encountered. These problems are well known and documented in the Ergonomics literature. Some studies have led to the questioning of the viability of road vehicle automation (Banks et al, 2018a; Stanton, 2015).

Research into Ergonomics aspects of driving automation began in the early 1990s. Initially, the research sought to understand the lessons that might be learnt from analogous domains, such as aviation (Stanton and Marsden, 1996). These lessons were: shortfalls in expected benefits, problems with equipment reliability, problems with driver skills maintenance, and error-inducing equipment designs. Over the past twenty years all of these predicted shortfalls have come to fruition. This has been shown most recently in the Tesla collision with the semi-trailer (Banks et al, 2018b) and the Uber collision with the cyclist. In one of the earliest published papers on vehicle automation, automated driving was show to both dramatically reduced workload and the readiness for the driver to intervene in emergencies (Stanton, Young and McCaulder, 1997). This study has been replicated many times over the past two decades with the same findings (e.g., Stanton et al, 2001; Stanton and Young, 2005; Young and Stanton, 2007; Stanton et al, 2011; de Winter et al, 2016; Eriksson and Stanton, 2017). A recent meta-analysis showed the effect of automated driving on mental workload to be very robust (de Winter et al, 2014).

The main factors associated with the driver in vehicle automation were initially identified by Stanton and Young (2000). These factors included: trust, situation awareness, mental workload, stress, locus of control, feedback, task demand and mental models. Many studies have investigated all of these factors over the years. The model was most recently updated by Heikoop et al (2016), which has extended the list of factors and interaction between factors. Young and Stanton $(2002,2004)$ in particular, investigated the effects of mental demand on the driver's attentional resources. They found that pool of attentional resources are dynamic and strongly yoked to task demand (called: Malleable Attentional Resources Theory: MART). This means that as task demand reduces (as it does with vehicle automation) then so does the driver's pool of attentional resources. The finding challenges the traditional notion of a fixed pool of attention and the concept that vehicle automation frees the driver up with spare capacity for monitoring the vehicle. The slow response by the driver to increased demand by the vehicle (as is the case with automation failure) is explained by the time it takes for the attentional resource pool to increase to the point where manual control can occur. Driver paced take-over of control of the vehicle by drivers has been found to be more successful than vehicle-paced (Eriksson and Stanton, 2018). Research is moving toward the sociotechnical approach to automation design, that seeks to develop both the technical and human aspects in a coevolutionary manner, rather than the traditional techno-centric approach (Banks et al, 2014; Banks and Stanton, 2016). The Society of Automotive Engineering have proposed 6 levels of driving automation though which we can consider this co-evolution: 
- Level 0: the driver is responsible for all aspects of driving, but the vehicle can provide automated warnings.

- Level 1: the driver must perform all driving tasks at any time, but is able to take advantage of limited automation for steering or acceleration and deceleration systems such as cruise control, lane keeping, and parking assistance systems.

- Level 2: the driver must monitor the automated system and be prepared to take control over at any time.

- Level 3: under limited conditions the driver is permitted to focus on tasks other than driving, but must be ready to take over when notified by the vehicle.

- Level 4: automated vehicle can safely operate in most scenarios, but requires the driver to determine when it is safe to do so. When vehicle automation is activated, the driver may place their attention elsewhere.

- Level 5: no human intervention except to start the system and provide a destination.

The purpose of this special issue is to report on the very latest research into driving automation and autonomy. There are seven papers that discuss aspects of the ergonomics issues as well as suggesting various ways forward for research and design.

\section{Ergonomics Issues in Driving Automation and Autonomy}

In the first paper, Kyriakidis et al (A human factors perspective on automated driving) interviewed 12 Human Factors scientists regarding their personal perspective on the challenges for levels 3 and 4 automated driving. All of the scientists agreed on the challenge of monitoring automation. This is not a task for which humans are well suited, and it is questionable whether or not drivers can regain control of a vehicle in a timely manner if automation drops out unexpectedly. There is a wealth of research in the Ergonomics literature that shows that humans do not perform well on extended vigilance tasks where there is an unpredictable requirement to intervene quickly. Concerns over trust in automation, driver mental workload and situation awareness featured heavily in the interviews, placing emphasis on driver training and interaction design in automated driving systems. Some of the scientists expressed the sentiment that unless driving automation and autonomy can completely replace the driver (i.e., levels 4 and 5 automation), then drivers should remain in the control loop. This is at odds with the approach being taken by vehicle manufacturers, which is to incrementally move up the automation levels, from 2 to 3, with aspirations for levels 4 and 5. Kyriakidis et al conclude that the main concern regarding vehicle automation is the ability of the driver to take-over control of the vehicle appropriate after a period of non-driving activities. It was argued that much might be learnt from aviation, as well as other automation domains.

In the second paper, Banks et al (Driving aviation forward: contrasting driving automation and aviation automation), argue that driving automation has already learnt many lessons from aviation (such as shortfalls in expected benefits, 
problems with equipment reliability, problems with driver skills maintenance, and error-inducing equipment designs). Given the rapid technological progress in driving automation, aviation has the prospect to also learn from the automotive domain. Both domains share similar goals of improving safety, efficiency, capacity and comfort, whilst simulateously reducing costs, emmisions, fuel consumption and congestion. Banks et al show that the role of the driver and pilot are conceptually similar (i.e., pilot-flying driver-driving, pilotmonitoring driver-monitoring, pilot-not-flying driver-not-driving). Similary, they consider the automation of operational, tactical and strategic tasks - noting that whilst early automation began with operational tasks it is moving up the hierarchy to tactical and strategic tasks. Banks et al debate the differing design philosophies in automation, such as the left over, compensatory and complementary principles. The latter of these principles seems to hold most promise for involving the human in shared control of the vehicle (albeit an automobile of aircraft). In an ideal situation, shared control capitalizes on the strengths and minimises the weakness of both human and technological agents in automated systems.

The third paper, Pampel et al (Getting the drivers back into the loop: the quality of manual vehicle control following long and short non-critical transfer of control requests) addresses the challenge of vehicle-to-driver hand-back of control in a driving simulator. The authors note that very few studies have investigated noncritical handovers, and none have addressed the time interval. Pampel et al systematically manipulated the transfer of control time, with a shorter time of 5 seconds and a longer time of 50 seconds. They anticipated that the in the longer handover time the driver would be better prepared for manual control (as they would have more time to build up their understanding of the road environment, behaviour of other vehicles as well as their own vehicle status). Pampel et al also manipulated the level of distraction. In one condition they were asked to monitor the road environment and in the other they were encouraged to interact with a game on a tablet computer. The finding from the study showed that drivers' manual control was better with the longer transfer of control time. As anticipated, the longer handover time enabled driver to be better prepared. These effects were diminished after a short period of manual control. Interestingly, the findings also suggest that engaging with the game on the tablet had a protective effect on mental resources. When playing with the game, rather than monitoring the road, drivers seemed better prepared to resume manual control (provided there was sufficient time to do so). This could be due to the MART effect, mentioned earlier.

In the fourth paper, Banks and Stanton (Analysis of driver roles: modeling the changing role of the driver in automated driving systems using EAST) model the effect that automation will have on the driver using the Event Analysis of Systemic Teamwork (EAST) method. EAST comprises three networks: social (communications structure and the communications taking place between the agents in the system), task (the relationships between tasks and their sequence and interdependences) and information (the information that the different agents use and communicate during task performance). These networks offer insights in the nature of distributed cognition in sociotechnical systems. 
Comparison of the task networks reveals that although there is only one additional task with automated driving, the nature of the driver's tasks (by assuming a monitoring role) have fundamentally changed. Comparison of the social networks show that they have increased dramatically. This increased in complexity is revealed by the social network metrics. The automated controller has become the dominant node in the network, over the driver. Comparison of the information networks shows that the number of nodes has increased by a quarter. Taken together, all of these network analyses point to the fact that the task on monitoring automation has increased, rather than reduced, the demand on the driver. EAST has provided both quantitative and qualitative insights into the effects of automation. Quantitative insights show the effects on the structural relationships between the driver, vehicle, environment and automation. Qualitative insights point out the changes in the nature of the driving tasks and information used. Both sets of insights reveal a cause for concern for the role of drivers monitoring vehicle automation.

In the fifth paper, Brell et al (Suspicious mind? Users' perceptions of autonomous and connected driving) undertook an interview study followed by a questionnaire study, to understand driver's preconceptions of autonomous driving and connected driving. These preconceptions were analyses separately for comparative purposes. The four main areas for consideration were: the need fro control, the need for privacy, risk taking and technical self-efficacy. Nineteen drivers participated in the interview study and 443 driver participated in the questionnaire study. From the interview study, it seemed that drivers are reluctant to give up manual control or their vehicles. The drivers also spoke about perceived issues with trust, privacy and data security. These concerns were further borne out in the questionnaire study, although differences between populations. Early adopters of technology were far more positive about the benefits, and less negative about the drawback, of autonomous and connected driving than traditionalists. In a summary of public perceptions of autonomous and connected driving, Brell et al show that safety was the primary concern, followed by the desire of the driver to maintain overall control of the vehicle.

The sixth paper, Cabrall et al (How to keep drivers engaged while supervising driving automation? A literature survey and categorization of six solution areas) proposed six potential solutions to the problem of driver engagement with vehicle automation, which are:

- Avoid the role of sustained human supervision of automation;

- Reduce the supervising role along an objective dimension;

- Reduce the supervising role along a subjective dimension;

- Support the supervising role from the behavioural paradigm;

- Support the supervising role from the dyadic cognitivism paradigm; and

- Support the supervising role from the triadic ecological paradigm.

There is a general concern that the new role of the driver with automated systems will lead to new types of problems and failures in driving. This could thwart the proposed benefits, such as reduced accidents, congestion and emissions. The problem of sustained human vigilance is well documented and is one of the biggest threats to vehicle automation. Cabrall et al review each of the six proposed solutions with reference back to the literature on automation. The 
relative merits are compared and contrasted. At present, there was no expressed preference for one strategy over another but they are not mutually exclusive options. Rather the six strategies were presented as thought experiments into the problem of vehicle automation and offer a way to classify studies as they emerge in the literature.

For the final paper, Navarro (A state of science on highly automated driving) reviews the literature on highly automated driving, to show how the subject area has grown over the past four decades. His search showed only one paper in the 1990 s, to three papers in the 2000s, to over one hundred in the 2010s. Navarro structured his review into four main areas: driver supervision, hand-back of control to the driver, control by automation, and the after-effects. In general, drivers tend to engage more with non-driving tasks when the vehicle is being controlled by automation. This effect has been found in driving simulators, on test tracks as well as on the open roads. Clearly there are safety implications if the driver is assumed to be in an automation-monitoring role. It does seem that there is a need to keep the driver busy in some way. Reclaiming manual control of the vehicle from automation in an emergency is an enduring concern. This has been found to be ineffective in many studies. Planned take over of control has faired better, provided enough time is given. Self-paced take over seems to perform best of all. Navarro's review suggests that drivers are reluctant to use automation and, when they do, it can have a negative transfer effect on their own driving style. He also reports negative effects on workload, situation awareness, trust, attention, distraction and fatigue. The review concludes with the notion that if automation is to replace human drivers, then they need to be kept busy with other activities. This makes them best placed to take control of the vehicle if manual control is required.

\section{Conclusions}

The field of driving automation and autonomy is fast moving and has gathered pace over the past four decades. Mush still remains unresolved however, so there is an urgent need to develop new paradigms and systems. As these systems are becoming commercial products, many of the predicted problems are being realized on the open roads (Banks et al, 2018a,b). Most of the research to date has been conducted in simulators, and there is good evidence that drivers behave in a similar manner in both environments (Eriksson et al, 2017).

The take-home messages from the papers in this thematic issue on driving automation and autonomy have been summarized as follows:

- The benefits of driving automation and autonomy have yet to be fully realized;

- MART explains why it takes time to get back into manual control;

- Concerns continue regarding the effects of automation on driver trust, mental workload, situation awareness, attention and fatigue;

- Driver do not perform well in period of extended vigilance (the so-called monitoring task);

- Performing a non-driving task can have a protective effect on attentional resources (helping reduce the take-over time);

- Driver-paced take over of control is better than vehicle-paced; 
- Longer take-over times can help with post take-over manual control stability;

- Concerns about emergency take-over are unresolved; and

- Driving automation and autonomy needs to be approached as coevolution using the sociotechnical systems paradigm.

At the beginning of this editorial it was suggested that the twin aims of driving automation and autonomy are to support drivers and solve societal problems. It is estimated that driving can account for six working weeks of the average driver's time. This time could productively be used for work, rest or leisure activities (Stanton, 2015). As yet, this has not been realised. The aim of this thematic issue is to stimulate research interest in this growing field of Ergonomics, and demonstrate some of what has been achieved to date. Clearly, much more need to be done before we can claim success for the goals of driving automation and autonomy.

\section{Acknowledgement}

This research was funded by the Engineering and Physical Sciences Research Council as part of the TASCC programme: Human Interaction: Designing Autonomy in Vehicles (HI:DAVe), grant reference number EP/N011899/1.

\section{References}

Banks, V.A., Eriksson, A., O'Donoghue, J. and Stanton, N.A. (2018a) Is partially automated driving a bad idea? Observations from an on-road study, Applied Ergonomics, 68, 138-145.

Banks, V. A., Plant, K. L. and Stanton, N. A. (2018b) Driver error or designer error: Using the Perceptual Cycle Model to explore the circumstances surrounding the fatal Tesla crash on 7th May 2016. Safety Science, 108, 278-285.

Banks, V.A. and Stanton, N.A. (2016) Keep the driver in control: Automating automobiles of the future. Applied Ergonomics, 53, 398-395.

Banks, V. A., Stanton, N. A. and Harvey, C. (2014) Sub-systems on the road to vehicle automation: Hands and feet free but not mind free driving. Safety Science, 62, 505-514.

de Winter, J. C. F., Happee, R., Martens, M. H. and Stanton, N. A. (2014) Effects of adaptive cruise control and highly automated driving on workload and situation awareness: a review of the empirical evidence. Transportation research part F: Traffic Psychology and Behaviour, 27, 196-217.

de Winter, J. C. F., Stanton, N. A., Price, J. S. and Mistry, H. (2016) The effects of driving with different levels of unreliable automation on self-reported workload and secondary task performance. International Journal of Vehicle Design, 70, (4) 297-324. 
Eriksson, A., Banks, V. and Stanton, N. A. (2017) Transition to Manual:

comparing simulator with on-road control transitions. Accident Analysis and Prevention, 102, 227-234.

Eriksson, A. and Stanton, N. A. (2017) Take-over time in highly automated vehicles: transitions to and from manual control. Human Factors, 59 (4), 689705 .

Eriksson, A. and Stanton, N. A. (2018) Driving Performance After Self-Regulated Control Transitions in Highly Automated Vehicles. Human Factors, 59(8), 12331248.

Heikoop, D. D., de Winter, J. C. F., van Arem, B. and Stanton, N. A. (2016) Psychological constructs in driving automation: a consensus model and critical comment on construct proliferation. Theoretical Issues in Ergonomics Science, 17 (3), 284-303.

Stanton, N. A. (2015) Responses to autonomous vehicles, Ingenia, 62, 9.

Stanton, N. A., Dunoyer, A. and Leatherland, A. (2011) Detection of new in-path targets by drivers using Stop \& Go Adaptive Cruise Control, Applied Ergonomics, 42 (4), 592-601.

Stanton, N. A. and Marsden, P. (1996) From fly-by-wire to drive-by-wire: safety implications of automation in vehicles. Safety Science, 24, (1) 35-49.

Stanton, N. A. and Young, M. (2000) A proposed psychological model of driving automation. Theoretical Issues in Ergonomics Science, 1 (4), 315-331.

Stanton, N. A. and Young, M. (2005) Driver behaviour with Adaptive Cruise Control. Ergonomics, 48, 1294 - 1313.

Stanton, N. A., Young, M. and McCaulder, B. (1997) Drive-by-wire: the case of mental workload and the ability of the driver to reclaim control. Safety Science, 27 (2-3), 149-159.

Stanton, N. A.; Young, M. S.; Walker, G. H.; Turner, H, and Randle, S.

(2001) Automating the driver's control tasks. International Journal of Cognitive Ergonomics, 5 (3), 221-236.

Young, M. S. and Stanton, N. A. (2002). Malleable Attentional Resources Theory: A new explanation for the effects of mental underload on performance. Human Factors, 44 (3), 365-375.

Young, M. S. and Stanton, N. A (2004) Taking the load off: investigations of how Adaptive Cruise Control affects mental workload. Ergonomics, 47 (8), 10141035. 
Young, M. S. and Stanton, N. A. (2007). Back to the future: Brake reaction times for manual and automated vehicles. Ergonomics, 50 (1), 46-58. 